\title{
PROOF OF A CONJECTURE OF RAMANUJAN
}

\author{
by A. O. L. ATKIN
}

(Received 11 February, 1966)

1. Introduction. We write

$$
f(x)=(1-x)\left(1-x^{2}\right)\left(1-x^{3}\right) \ldots
$$

and

$$
\sum_{n=0}^{\infty} p(n) x^{n}=1 / f(x)
$$

so that $p(n)$ is the number of unrestricted partitions of $n$. Ramanujan [1] conjectured in 1919 that if $q=5,7$, or 11 , and $24 m \equiv 1\left(\bmod q^{n}\right)$, then $p(m) \equiv 0\left(\bmod q^{n}\right)$. He proved his conecture for $n=1$ and $2 \uparrow$, but it was not until 1938 that Watson [4] proved the conjecture for $q=5$ and all $n$, and a suitably modified form for $q=7$ and all $n$. (Chowla [5] had previously observed that the conjecture failed for $q=7$ and $n=3$.) Watson's method of modular equations, while theoretically available for the case $q=11$, does not seem to be so in practice even with the help of present-day computers. Lehner $[6,7]$ has developed an essentially different method, which, while not as powerful as Watson's in the cases where $\Gamma_{0}(q)$ has genus zero, is applicable in principle to all primes $q$ without prohibitive calculation. In particular he proved the conjecture for $q=11$ and $n=3$ in [7]. Here I shall prove the conjecture for $q=11$ and all $n$, following Lehner's approach rather than Watson's. I also prove the analogous and essentially simpler result for $c(m)$, the Fourier coefficient $\ddagger$ of Klein’s modular invariant $j(\tau)$ as

THEOREM 1. If $m \equiv 0\left(\bmod 11^{n}\right)$, then $c(m) \equiv 0\left(\bmod 11^{n}\right)$.

The full truth with regard to Ramanujan's original conjecture is thus now known to be: If $24 m \equiv 1\left(\bmod 5^{a} 7^{b} 11^{c}\right)$, then $p(m) \equiv 0\left(\bmod 5^{a} 7^{\beta} 11^{c}\right)$, where $\beta=[(b+2) / 2]$.

In view of Watson's result we need only prove here

THEOREM 2. If $24 m \equiv 1\left(\bmod 11^{n}\right)$, then $p(m) \equiv 0\left(\bmod 11^{n}\right)$.

The general plan of the paper is as follows. In $\S 2$ we describe the notation and general theory required for the proof of Theorem 1 . In $\S 3$ we carry through sufficient detailed calculation to prove Theorem 1. In $\S 4$ the additional theory required for the proof of Theorem 2 is given, and in $\S 5$ Theorem 2 is proved. Necessary calculations which would unduly interrupt the main argument are given in Appendices.

\section{Functions on $\Gamma_{0}(11)$.}

2.1. We consider the subgroup $\Gamma_{0}(11)$ of the full modular group $\Gamma(1)$, defined by those transformations

$$
\tau \rightarrow V \tau=\frac{a \tau+b}{c \tau+d} \quad(a, b, c, d, \text { integral with } a d-b c=1)
$$

† Ramanujan [1, 2]. See also Rushforth [3].

$\ddagger$ We take the Fourier series of $j(\tau)$ with leading coefficient unity and constant term zero. Thus $j(\tau)=$ $x^{-1}+196884 x+\ldots$ with $x=e^{2 \pi i \tau}$. 
of $\Gamma(1)$ that satisfy $c \equiv 0(\bmod 11) . \Gamma_{0}(11)$ is of genus 1 , and its fundamental region has two cusps $\tau=i \infty$ and $\tau=0$, with local variables $x=e^{2 \pi i \tau}, x=e^{-2 \pi i / 11 \tau}$, respectively. By "an entire modular function on $\Gamma_{0}(11)$ " we understand a function $F(\tau)$, regular in $\operatorname{Im} \tau>0$, that satisfies $F(V \tau)=F(\tau)$ for $V \in \Gamma_{0}(11)$, and has at most polar singularities in the local variables at the two cusps of $\Gamma_{0}(11)$. For such $F(\tau)$ we shall write $F \in S$. If in addition $F(\tau)$ is zero at $\tau=i \infty 0$ we write $F \in S^{\infty}$. Finally, if $F(\tau)$ is zero at $\tau=0$ we write $F \in S^{0}$.

We refer to the expansion of $F(\tau)$ in powers of $x=e^{2 \pi i \tau}$ at $\tau=i \infty$ as its Fourier series (FS).

We have:

Lemma 1. If $F(\tau) \in S$, then $F^{*}(\tau)=F(-1 / 11 \tau) \in S$.

A simple proof is given by Newman [9, Lemma 1]. It is clear that the expansion of $F(\tau)$ at $\tau=0$ is the FS of $F^{*}(\tau)$, and that $F \in S^{\infty}, S^{0} \Leftrightarrow F^{*} \in S^{0}, S^{\infty}$.

We now introduce a linear operator $U$ defined by

$$
\begin{aligned}
11 U F(\tau) & =\sum_{r=0}^{10} F\left(\frac{\tau+r}{11}\right), \\
U^{n+1} F(\tau) & =U\left(U^{n} F(\tau)\right) \quad(n \geqq 1) .
\end{aligned}
$$

Clearly

$$
U\left(a_{1} F_{1}+a_{2} F_{2}\right)=a_{1} U F_{1}+a_{2} U F_{2},
$$

if $a_{1}, a_{2}$ are constants. If the FS of $F(\tau)$ is

$$
\sum_{r=r_{0}}^{\infty} \alpha_{r} x^{r}
$$

then the FS of $U F(\tau)$ is

$$
\sum_{11 r \geq r_{0}} \alpha_{11} x^{r}
$$

By $U F(-1 / 11 \tau)$ we shall understand the effect of replacing $\tau$ by $-1 / 11 \tau$ in $U F(\tau)$ and not " $U G(\tau)$ where $G(\tau)=F(-1 / 11 \tau)$ ".

We also write

$$
F_{1}(\tau) \equiv F_{2}(\tau)(\bmod m)
$$

if all the respective coefficients in the FS of $F_{1}(\tau)$ and $F_{2}(\tau)$ are congruent modulo $m$. Thus nothing is asserted by (2) as to the expansions at $\tau=0$.

It will be convenient in the sequel to assess divisibility by powers of 11 by using an exponential valuation. Accordingly, for integral $a$, we define $\pi(a)$ by

$$
11^{\pi(a)} \mid a, \quad 11^{\pi(a)+1} \nmid a,
$$

and for rational $a=b / c$ we define

$$
\pi(a)=\pi(b)-\pi(c) .
$$

We write conventionally $\pi(0)=\infty$, and regard any inequality $\pi(0) \geqq k$ as valid. 
We have

$$
\begin{aligned}
\pi(a b) & =\pi(a)+\pi(b), \\
\pi(a+b) & \geqq \min (\pi(a), \pi(b)),
\end{aligned}
$$

with equality if $\pi(a) \neq \pi(b)$. follows.

The crucial results on $U F(\tau)$ are given by Lehner (Theorem 8 and (8.81) of [6]), and are as

LEMMA 2. If $F(\tau) \in S$, then

$$
\text { (i) } U F(\tau) \in S \text {, (ii) } 11 U F(-1 / 11 \tau)-11 U F(11 \tau)=F(-1 / 121 \tau)-F(\tau) \text {. }
$$

Note that in (ii) $U F(11 \tau)$ and $F(-1 / 121 \tau)$ are not themselves in $S$. It is also immediate that

$$
F(\tau) \in S^{\infty} \Rightarrow U F(\tau) \in S^{\infty}
$$

((4) is not valid for $S_{0}$.)

Reverting now to the proof of Theorem 1, we see that, since $j(\tau) \in S$, then $U^{n} j(\tau) \in S^{\infty}$ for $n \geqq 1$. Theorem 1 is then equivalent to proving that the FS of $11^{-n} U^{n} j(\tau)$ has integral coefficients. To establish this, we obtain first a standard basis for the functions of $S^{\infty}$, and then use Lemma 2 to obtain detailed information as to the effect of the operator $U$ on these functions.

2.2. A linear basis for functions on $\Gamma_{0}(11)$. The following lemma is proved in Appendix $A$.

LEMMA 3. For all integral $n \geqq 2$, there exist functions $G_{n}(\tau), g_{n}(\tau), h_{n}(\tau)$ with the following properties:

$$
\begin{aligned}
G_{n}(\tau) \in S^{0}, g_{n}(\tau) \in S^{\infty}, h_{n}(\tau) \in S^{\infty}, \\
\begin{aligned}
G_{n}(-1 / 11 \tau) & =h_{n}(\tau)=11^{\theta(n)} g_{n}(\tau), \\
\theta(n) & =6 k+2,3,4,6,6 \\
n & =5 k+2,3,4,5,6 \quad(k \geqq 0) .
\end{aligned}
\end{aligned}
$$

where

according as

(iii) The FS of $G_{n}(\tau)$ has integral coefficients with leading term $x^{-n}$.

(iv) The FS of $g_{n}(\tau)$ has integral coefficients with leading term $x^{\psi(n)}$,

where

$$
\begin{aligned}
\psi(n) & =5 k+1,2,3,5,4 \\
n & =5 k+2,3,4,5,6 \quad(k \geqq 0) .
\end{aligned}
$$

Further, there exists a function $B(\tau) \in S$ with simple poles at $\tau=0$ and $\tau=i \infty$, such that $B(-1 / 11 \tau)=B(\tau)$. The FS of $B(\tau)$ has integral coefficients, with leading term $x^{-1}$.

Since the Riemann surface of $\Gamma_{0}(11)$ cannot support a univalent function, we have the immediate corollary: 
LEMMA 4. Suppose that $F(\tau) \in S$ has a pole of order $M$ at $\tau=0$ and a pole of order $N$ at $\tau=i \infty$. Then

$$
F(\tau)=\sum_{r=2}^{N} \lambda_{-r} G_{r}(\tau)+\lambda_{-1} B(\tau)+\lambda_{0}+\sum_{r=2}^{M} \lambda_{r} h_{r}(\tau),
$$

where the $\lambda_{r}(-N \leqq r \leqq M)$ are constants.

Finally we restate Lemma 4 in the case of greatest interest to us.

Lemma 5. Suppose that $F(\tau) \in S^{\infty}$ has a pole of order $M$ at $\tau=0$. Then

$$
F(\tau)=\sum_{r=2}^{M} \lambda_{r} h_{r}(\tau), \quad F(-1 / 11 \tau)=\sum_{r=2}^{M} \lambda_{r} G_{r}(\tau)
$$

For a given $F(\tau) \in S^{\infty}$, the constants $\lambda_{r}$ in Lemma 5 can be determined from the FS of either $F(-1 / 11 \tau)$ or $F(\tau)$. We are mainly concerned not with the exact value of $\lambda_{r}$, but with $\pi\left(\lambda_{r}\right)$. In $\S 3$ below we obtain suitable lower bounds for $\pi\left(\lambda_{r}\right)$ in the case when $F(\tau)=$ $U g_{n}(\tau)$. The calculations take a simpler form when we consider $F(\tau)=U h_{n}(\tau)$; the transition to $U g_{n}(\tau)$ is immediate from Lemma 3(ii).

3.1. Since $h_{n}(\tau) \in S^{\infty}$, we have

$$
11 U h_{n}(\tau)=\sum c_{n r} h_{r}(\tau)
$$

where the $c_{n r}$ are constants, by Lemma 5 . It is convenient to regard the sum in (5) as one from $r=2$ to $\infty$, although all but a finite number of the $c_{n r}$ are zero. We have also

$$
11 U h_{n}(-1 / 11 \tau)=\sum c_{n r} G_{r}(\tau)
$$

and, by Lemma 2(ii),

$$
11 U h_{n}(-1 / 11 \tau)-11 U h_{n}(11 \tau)=-h_{n}(\tau)+h_{n}(-1 / 121 \tau) .
$$

It follows from (7) that the principal part of the FS of $11 U h_{n}(-1 / 11 \tau)$ is the same as that of $h_{n}(-1 / 121 \tau)=G_{n}(11 \tau)$, since $U h_{n}(11 \tau)$ and $h_{n}(\tau)$ are zero at $\tau=i \infty$. Hence the coefficients $c_{n r}$ may be uniquely determined by the fact that the FS of

$$
G_{n}(11 \tau)-\sum c_{n r} G_{r}(\tau)
$$

has no terms in $x^{-11 n}, \ldots, x^{-3}, x^{-2}$. It will then necessarily have no term in $x^{-1}$, which provides a check in numerical work. It follows that the $c_{n r}$ are integers (since each $G_{r}$ has leading term $x^{-r}$, and the FS of $G_{n}(11 \tau)$ has integral coefficients) and also that

$$
c_{n r}=0 \text { if } r>11 n \text {. }
$$

Considering next the determination of the $c_{n r}$ from (5) we observe that for different $r$ the FS of $h_{r}(\tau)$ commence with different powers $\dagger$ of $x$, by Lemma 3(iv).

† The linear basis used by Lehner $[6,7]$ does not have this property.

B 
Thus, since every coefficient in the FS of $11 U h_{n}(\tau)$ is divisible by $11^{\theta(n)+1}$, and the leading term in the FS of $h_{r}(\tau)$ is $11^{\theta(r)} x^{\psi(r)}$, we have

and

$$
\pi\left(c_{n r}\right) \geqq \theta(n)-\theta(r)+1
$$

We now establish certain conditions under which $\pi\left(c_{n r}\right) \geqq 3$.

3.2. The values of $c_{n r}\left(\bmod 11^{3}\right)$. All congruences in this section are to the modulus $11^{3}$. It follows from (6) and (7) that

$$
G_{2}(11 \tau)-11^{2} g_{2}(\tau) \equiv \sum c_{2 r} G_{r}(\tau), \quad G_{n}(11 \tau) \equiv \sum c_{n r} G_{r}(\tau) \quad(n \geqq 3) .
$$

We shall use the symbol $(k, l, m)$ to denote an expression of the form

$$
11^{2} \sum_{i=k}^{l-1} \lambda_{i} G_{i}(\tau)+11 \sum_{i=l}^{m-1} \lambda_{i} G_{i}(\tau)+\sum_{i=m}^{N} \lambda_{i} G_{i}(\tau),
$$

where $k<l<m \leqq N$ and the $\lambda_{l}$ are integral constants. Then by direct calculation $\dagger$ we find

$$
G_{2}(11 \tau)-11^{2} g_{2}(\tau) \equiv(2,9,19), \quad G_{3}(11 \tau) \equiv(8,18,28) .
$$

Now Table 5 in Appendix B shows that

$$
G_{i}(\tau) G_{j}(\tau)=\sum_{r=-3}^{0} \mu_{r} G_{i+j+r}(\tau)
$$

where the $\mu_{r}$ are integral, and $\mu_{-3}, \mu_{-2} \equiv 0(\bmod 11)$, for all $i$ and $j$. It follows that

where

$$
\left(k_{1}, l_{1}, m_{1}\right)\left(k_{2}, l_{2}, m_{2}\right) \equiv\left(k_{3}, l_{3}, m_{3}\right),
$$

$$
\begin{aligned}
k_{3} & =\min \left(k_{1}+m_{2}-1, k_{2}+m_{1}-1, l_{1}+l_{2}-1\right), \\
l_{3} & =\min \left(l_{1}+m_{2}-1, l_{2}+m_{1}-1\right), \\
m_{3} & =m_{1}+m_{2}-1 .
\end{aligned}
$$

Further, from Table 6 we have, for $m \geqq 4$,

$$
11^{2} g_{2}(\tau) .(k, l, m) \equiv(m-2, \infty, \infty)
$$

Thus

$$
\begin{aligned}
& G_{4}(11 \tau)=G_{2}^{2}(11 \tau)-11 G_{3}(11 \tau) \equiv(17,27,37), \\
& G_{5}(11 \tau)=G_{2}(11 \tau) G_{3}(11 \tau)-11 G_{4}(11 \tau) \equiv(26,36,46) \\
& G_{6}(11 \tau)=G_{2}(11 \tau) G_{4}(11 \tau) \equiv(35,45,55) \\
& G_{7}(11 \tau)=G_{2}(11 \tau) G_{5}(11 \tau) \equiv(44,54,64)
\end{aligned}
$$

† This was done in three different ways on three different machines: firstly using Lemma 9 on a Diehl desk calculator at Durham University; next using (5) on an Elliott 803 computer at Durham University; and finally using (6) on the I.C.T. Atlas 1 computer at Chilton. The computing times were respectively one week, one hour, and ten seconds. 
It is now easily seen by induction, since $G_{n+5}(\tau)=G_{n}(\tau) G_{5}(\tau)$, that

$$
G_{n}(11 \tau) \equiv(9 n-19,9 n-9,9 n+1) \quad(n \geqq 3),
$$

and thus, by (11),

$$
\pi\left(c_{n r}\right) \geqq 3 \text { if } r \leqq 9 n-20, n \geqq 3
$$

3.3. The values of $c_{n r}\left(\bmod 11^{4}\right)$. We have $11 U h_{n}(\tau) \equiv 0\left(\bmod 11^{4}\right)$ for $n \geqq 3($ since $\theta(n)+1 \geqq 4)$, and $(5)$ with $\pi\left(c_{21}\right) \geqq 2, \pi\left(c_{22}\right) \geqq 1$ gives $11 U h_{2}(11 \tau) \equiv 0\left(\bmod 11^{4}\right)$.

Then, by (6) and (7),

$$
\begin{aligned}
& G_{2}(11 \tau)-11^{2} g_{2}(\tau) \equiv \sum c_{2 r} G_{r}(\tau)\left(\bmod 11^{4}\right), \\
& G_{3}(11 \tau)-11^{2} g_{3}(\tau) \equiv \sum c_{3 r} G_{r}(\tau)\left(\bmod 11^{4}\right), \\
& G_{n}(11 \tau) \equiv \sum c_{n r} G_{r}(\tau) \quad\left(\bmod 11^{4}\right)(n \geqq 4) .
\end{aligned}
$$

Hence, by arguments similar to those of $\S 3.2$, we obtain

$$
c_{5 r} \equiv 0\left(\bmod 11^{4}\right) \text { for } r \leqq 15, c_{7 r} \equiv 0\left(\bmod 11^{4}\right) \text { for } r \leqq 33 .
$$

A crude induction, using Table 5 and (15), now shows that

$$
c_{5 k+2, r} \equiv 0\left(\bmod 11^{4}\right) \text { for } r \leqq 15 k+18, k \geqq 1 .
$$

We summarise our results on $c_{n r}$ in the forms actually required later.

LEMMA 6.

$$
\begin{array}{llr}
\pi\left(c_{n r}\right) \geqq 0 & \text { always, } & \text { (from §3.1) } \\
\pi\left(c_{n r}\right) \geqq \theta(n)-\theta(r)+1 & \text { always, } & \text { (from (9)) } \\
\pi\left(c_{n r}\right) \geqq 1 & \text { for } n=2 \text { or } 3,9 \leqq r \leqq 11, & \text { (from (12)) } \\
\pi\left(c_{n r}\right) \geqq 2 & \text { for } n=2 \text { or } 3, r \leqq 8, & \text { (from (12)) } \\
\pi\left(c_{n r}\right) \geqq 3 & \text { for } n=4, r \leqq 16, & \text { (from (15)) } \\
\pi\left(c_{n r}\right) \geqq 3 & \text { for } n \geqq 5, r \leqq n+14, & \text { (from (15)) } \\
\pi\left(c_{n r}\right) \geqq 4 & \text { for } n \equiv 2(\bmod 5), n \geqq 7, r=n-1 \text { or } n-2, & \text { (from (17)) } \\
\pi\left(c_{22}\right)=2, \pi\left(c_{32}\right)=3, \pi\left(c_{42}\right)=4 . &
\end{array}
$$

3.4. We now use the results of $\S 3.3$ to show that, in effect, functions of a suitable form remain of that form under the operation $11^{-1} U$. This is the basis of the proofs of Theorems

$\dagger$ This result is by no means best possible. We can, by consideration of cases (mod 5), establish results with $11 n$ instead of $9 n$ on the right-hand side of (14), but (15) suffices later. 
1 and 2. Our Lemma 7 below is needed in $\S 5$, although a weaker form would suffice for Theorem 1.

We define

and

$$
\xi(2)=0, \xi(3)=1
$$

according as

$$
\xi(n)=5 k+1,3,3,4,5
$$

We also define $\eta(2)=0, \eta(3)=1, \eta(n)=\xi(n)+1(n \geqq 4)$. We shall denote by $X$ the class of functions $F(\tau)$ with

$$
F(\tau)=\sum_{n=2}^{N} \lambda_{n} 11^{\xi(n)} g_{n}(\tau),
$$

and by $Y$ the class of functions $F(\tau)$ with

$$
F(\tau)=\sum_{n=2}^{M} \mu_{n} 11^{\eta(n)} g_{n}(\tau),
$$

where $N, M, \lambda_{n}$, and $\mu_{n}$ are any integral constants.

LEMMA 7. If $F(\tau) \in X$, then $11^{-1} U F(\tau) \in Y$.

Proof. We have, by (5),

$$
11^{-1} U \sum \lambda_{n} 11^{\xi(n)} g_{n}(\tau)=\sum \sum \lambda_{n} 11^{\xi(n)-2-\theta(n)+\theta(r)} c_{n r} g_{r}(\tau) .
$$

Thus we have to show that, for all $n$ and $r$,

$$
\xi(n)-2-\theta(n)+\theta(r)+\pi\left(c_{n r}\right) \geqq \eta(r) .
$$

The following table is given to clarify the details of the proof $(k \geqq 0)$.

\begin{tabular}{|l|cc|ccccc|cc|}
\hline$n$ & 2 & 3 & $5 k+4$ & $5 k+5$ & $5 k+6$ & $5 k+7$ & $5 k+8$ & $5 k+9$ & $5 k+10$ \\
$\xi(n)$ & 0 & 1 & $5 k+1$ & $5 k+3$ & $5 k+3$ & $5 k+4$ & $5 k+5$ & $5 k+6$ & $5 k+8$ \\
$\eta(n)$ & 0 & 1 & $5 k+2$ & $5 k+4$ & $5 k+4$ & $5 k+5$ & $5 k+6$ & $5 k+7$ & $5 k+9$ \\
$\theta(n)$ & 2 & 3 & $6 k+4$ & $6 k+6$ & $6 k+6$ & $6 k+8$ & $6 k+9$ & $6 k+10$ & $6 k+12$ \\
$\theta(n)-\eta(n)$ & 2 & 2 & $k+2$ & $k+2$ & $k+2$ & $k+3$ & $k+3$ & $k+3$ & $k+3$ \\
$\theta(n)-\xi(n)$ & 2 & 2 & $k+3$ & $k+3$ & $k+3$ & $k+4$ & $k+4$ & $k+4$ & $k+4$ \\
\hline
\end{tabular}

We quote the results of Lemma 6 without their formula numbers. Since $\pi\left(c_{n r}\right) \geqq 0,(21)$ holds if $\theta(r)-\eta(r) \geqq \theta(n)-\xi(n)+2$. This is satisfied in the cases

$$
n \geqq 5, r \geqq n+15 ; n=4, r \geqq 17 ; n=2 \text { or } 3, r \geqq 12 \text {. }
$$


Also, since $\pi\left(c_{n r}\right) \geqq \theta(n)-\theta(r)+1$, (21) holds if $\xi(n) \geqq \eta(r)+1$. This is satisfied in the cases

$$
n \geqq 5, n \not \equiv 2(\bmod 5), \quad r \leqq n-2 ; n \geqq 5, n \equiv 2(\bmod 5), r \leqq n-3 .
$$

Now for $n \geqq 5, n-1 \leqq r \leqq n+14$, we have $\pi\left(c_{n r}\right) \geqq 3$, and so (21) holds if

$$
\theta(n)-\xi(n) \leqq \theta(r)-\eta(r)+1 \text {. }
$$

This is valid unless $n \equiv 2(\bmod 5), r=n-1$. This gives the cases

$$
n \geqq 5, n \not \equiv 2(\bmod 5), n-1 \leqq r \leqq n+14 ; n \geqq 5, n \equiv 2(\bmod 5), r \geqq n .
$$

Next, if $n \geqq 5, n \equiv 2(\bmod 5), r=n-1$ or $n-2$, we have $\pi\left(c_{n r}\right) \geqq 4$. Hence (21) holds if

$$
n \geqq 5, n \equiv 2 \quad(\bmod 5), \quad r=n-1 \text { or } n-2 .
$$

Now for $n=4$, (21) is $\pi\left(c_{4 r}\right) \geqq \eta(r)-\theta(r)+5$. For $2 \leqq r \leqq 16$, we have $\pi\left(c_{4 r}\right) \geqq 3$, $\eta(r)-\theta(r) \leqq-2$. This gives

$$
n=4, \quad 2 \leqq r \leqq 16 .
$$

Finally if $n=2$ or 3 , (21) is $\pi\left(c_{n r}\right) \geqq \eta(r)-\theta(r)+4$, and we have for $r \leqq 8, \pi\left(c_{n r}\right) \geqq 2$ and $\eta(r)-\theta(r) \leqq-2$; also for $9 \leqq r \leqq 11$ we have $\pi\left(c_{n r}\right) \geqq 1$ and $\eta(r)-\theta(r) \leqq-3$. This gives

$$
n=2 \text { or } 3, \quad r \leqq 11 \text {. }
$$

Since (22) to (27) cover all integral $n, r$ with $n \geqq 2$ and $r \geqq 2$, Lemma 7 is proved.

COROLLARY. If $F(\tau) \in X$, then

$$
11^{-1} U F(\tau) \in X
$$

For $\xi(n) \leqq \eta(n)$.

It is desirable in some cases to prove that the congruences obtained by using Lemma 7 are best possible. To this end we define classes of functions $X^{0}$ and $Y^{0}$ as at the beginning of this section, but with the additional conditions $\pi\left(\lambda_{2}\right)=0, \pi\left(\mu_{2}\right)=0$. We now prove

LeMma 8. If $F(\tau) \in X^{0}$, then $11^{-1} U F(\tau) \in Y^{0}$.

We have, as in the proof of Lemma 7 ,

$$
\mu_{2}=\sum \lambda_{n} 11^{\xi(n)-2-\theta(n)+2} c_{n 2}=\sum \rho_{n}, \text { say. }
$$

Now $\pi\left(c_{n 2}\right) \geqq \theta(n)-\theta(2)+1$, so that, for $n \geqq 5$, we have $\pi\left(\rho_{n}\right) \geqq 1$, since $\xi(n) \geqq 2$. For $n=3$, $\pi\left(c_{32}\right)=3, \xi(3)-\theta(3)=-2$ and so $\pi\left(\rho_{3}\right) \geqq 1$. For $n=4, \pi\left(c_{42}\right)=4, \xi(4)-\theta(4)=-3$, and so $\pi\left(\rho_{4}\right) \geqq 1$. Hence

$$
\mu_{2} \equiv \hat{\lambda}_{2} 11^{-2} c_{22}(\bmod 11) \text {. }
$$

But $\pi\left(c_{22}\right)=2$, and hence, if $\pi\left(\lambda_{2}\right)=0$, then $\pi\left(\mu_{2}\right)=0$. This proves the lemma. 


\subsection{Proof of Theorem 1.}

We may express Lemma 2 (ii), in the form:

LEMMA 9. If $F(\tau) \in S$, then $F(-1 / 11 \tau)+11 U F(\tau)$ is an entire function on the full modular group $\Gamma(1)$.

Choosing $F(\tau)=B(\tau)$, we have, since the FS of $B(\tau)$ is $x^{-1}-5+\ldots$,

$$
60+B(\tau)+11 U B(\tau)=j(\tau) .
$$

Now $U\{B(\tau)+5\} \in S^{\infty}$, and $11 U B(-1 / 11 \tau)$ has FS $x^{-11}+O\left(x^{-1}\right)$. Hence

$$
11\{U B(-1 / 11 \tau)+5\}=\sum_{n=2}^{11} \alpha_{n} G_{n}(\tau),
$$

where the $\alpha_{n}$ are integral constants, and so

$$
11^{-1}\{U B(\tau)+5\}=\sum_{n=2}^{11} \alpha_{n} 11^{\theta(n)-2} g_{n}(\tau) \in X
$$

Thus

$$
11^{-1} U j(\tau)=11^{-1} U\{B(\tau)+5\}+U^{2}\{B(\tau)+5\} \in X,
$$

by (28). Repeated application of (28) shows that $11^{-n} U^{n} j(\tau) \in X$ for $n \geqq 1$.

Now the FS of any function in $X$ has integral coefficients, while the FS of $U^{n} j(\tau)$ is $\sum_{m=1}^{\infty} c\left(11^{n} m\right) x^{m}$. Hence for all $m \geqq 1, n \geqq 1$ we have that $11^{-n} c\left(11^{n} m\right)$ is an integer, which is Theorem 1.

Theorem 1 is best possible in the sense that $c\left(11^{n}\right) \neq 0\left(\bmod 11^{n+1}\right)$. We have

$$
11^{-1} U j(\tau) \equiv \alpha_{2} g_{2}(\tau)(\bmod 11) .
$$

Now $\alpha_{2}=1627$ and so $\pi\left(\alpha_{2}\right)=0$. Hence, by repeated application of Lemma 8 , we have (since $Y^{0} \subseteq X^{0}$ )

$$
11^{-n} U^{n} j(\tau) \in X^{0} \text {, }
$$

and so

$$
11^{-n} U^{n} j(\tau) \equiv k_{n} g_{2}(\tau)(\bmod 11),
$$

where $\pi\left(k_{n}\right)=0$.

Thus

$$
11^{-n} c\left(11^{n}\right) \equiv k_{n} \quad(\bmod 11),
$$

and so

$$
c\left(11^{n}\right) \not \equiv 0\left(\bmod 11^{n+1}\right) \text {. }
$$

4.1. We now define

$$
\eta(\tau)=e^{\pi i \tau / 12} f(x) \quad(\operatorname{In} \tau>0)
$$

where

$$
f(x)=\prod_{r=1}^{\infty}\left(1-x^{r}\right) \text { and } x=e^{2 \pi i \tau}
$$

and

$$
\phi(\tau)=\eta(121 \tau) / \eta(\tau)=x^{5} f\left(x^{121}\right) / f(x), \quad \Phi(\tau)=1 / \phi(\tau) .
$$


We also let

$$
\left.\begin{array}{rlrl}
l_{2 n-1} & =\left(13.11^{2 n-1}+1\right) / 24 & & (n \geqq 1), \\
l_{2 n} & =\left(23.11^{2 n}+1\right) / 24 & & (n \geqq 1),
\end{array}\right\}
$$

so that $l_{n}$ is the least positive integral solution of $24 l_{n} \equiv 1\left(\bmod 11^{n}\right)$.

Further let

$$
\left.\begin{array}{c}
\Lambda_{2 n-1}(x)=f\left(x^{11}\right) \sum_{m=0}^{\infty} p\left(11^{2 n-1} m+l_{2 n-1}\right) x^{m+1}, \\
\Lambda_{2 n}(x)=f(x) \sum_{m=0}^{\infty} p\left(11^{2 n} m+l_{2 n}\right) x^{m+1},
\end{array}\right\} \quad(n \geqq 1)
$$

and define a sequence of functions $L_{n}(\tau)$ by

$$
\left.\begin{array}{rlrl}
L_{1}(\tau) & =U \phi(\tau), & & \\
L_{2 n}(\tau) & =U L_{2 n-1}(\tau) & & (n \geqq 1), \\
L_{2 n+1}(\tau) & =\left\{U \phi\left(\tau L_{2 n}(\tau)\right\}\right. & & (n \geqq 1) .
\end{array}\right\}
$$

We shall prove by induction that, for $n \geqq 1, \Lambda_{n}(x)$ is the FS of $L_{n}(\tau)$. We have

$$
U\left\{F_{1}(11 \tau) F_{2}(\tau)\right\}=F_{1}(\tau) U F_{2}(\tau) .
$$

Now the FS of $\phi(\tau)$ is

$$
x^{5} f\left(x^{121}\right) \sum_{m=0}^{\infty} p(m) x^{m}
$$

so that the FS of $U \phi(\tau)$ is

$$
f\left(x^{11}\right) \sum_{m=0}^{\infty} p(11 m+6) x^{m+1}=\Lambda_{1}(x)
$$

Assuming that the FS of $L_{2 n-1}(\tau)$ is $\Lambda_{2 n-1}(x)$, we see that the FS of $L_{2 n}(\tau)=U L_{2 n-1}(\tau)$ is

$$
f(x) \sum_{m=0}^{\infty} p\left\{11^{2 n-1}(11 m+10)+l_{2 n-1}\right\} x^{m+1}=L_{2 n}(x) .
$$

Finally if the FS of $L_{2 n}(\tau)$ is $\Lambda_{2 n}(x)$, then the FS of $L_{2 n+1}(\tau)=U\left\{\phi(\tau) L_{2 n}(\tau)\right\}$ is

$$
f\left(x^{11}\right) \sum_{m=0}^{\infty} p\left\{11^{2 n}(11 m+6)+l_{2 n}\right\} x^{m+1}=L_{2 n+1}(x) .
$$

Since the expansions of $1 / f(x)$ and $1 / f\left(x^{11}\right)$ have integral coefficients with leading terms unity, Theorem 2 is equivalent to

LEMMA 10. The FS of $11^{-n} L_{n}(\tau)$ has integral coefficients. 
4.2. Since $\phi(\tau)$ is not a function on $\Gamma_{0}(11)$, but on $\Gamma_{0}(121)$, we cannot apply the methods of $\$ 2$ and 3 immediately. However we do have

LEMMA 11. If $F(\tau) \in S^{\infty}$, then

(i) $U\{\phi(\tau) F(\tau)\} \in S^{\infty}$,

(ii) the principal part of the expansions of $U\{\phi(\tau) F(\tau)\}$ in powers of $x=e^{-2 \pi i / 11 \tau}$ at its pole $\tau=0$ is the same as the principal part of the FS expansion of $11^{-2} \Phi(\tau) F(-1 / 121 \tau)$ in powers of $x=e^{2 \pi i \tau}$.

Further (i) and (ii) hold in the special case $F(\tau)=1$.

Lemma 11 is proved by Lehner [6, Theorem 8]; there are some misprints corrected in Lehner [7, page 178].

We now have, by Lemma 5 ,

$$
11^{2} U\left\{\phi(\tau) h_{n}(\tau)\right\}=\sum d_{n r} h_{r}(\tau),
$$

where the $d_{n r}$ are constants, and in fact zero if $11 \psi(r)<\psi(n)+5$ or $r>11 n+5$. Further the $d_{n r}$ are uniquely determined by the fact that the FS of

$$
\Phi(\tau) G_{n}(11 \tau)-\sum_{r=2}^{11 n+5} d_{n r} G_{r}(\tau)
$$

has no terms in $x^{-11 n-5}, \ldots, x^{-3}, x^{-2}$. Hence

$$
\pi\left(d_{n r}\right) \geqq 0 \text {. }
$$

We have also, from (34),

and thus

$$
11^{2+\theta(n)} U\left\{\phi(\tau) g_{n}(\tau)\right\}=\sum d_{n r} 11^{\theta(r)} g_{r}(\tau),
$$

$$
\pi\left(d_{n r}\right) \geqq \theta(n)-\theta(r)+2 \text {. }
$$

We could, by using

$$
\Phi(\tau) G_{n}(11 \tau) \equiv G_{5}(\tau)\left\{G_{n}(\tau)\right\}^{11}(\bmod 11),
$$

obtain quite easily conditions under which $\pi\left(d_{n r}\right) \geqq 1$. Unfortunately this is not quite enough to prove Theorem 1 , and we require the following

LEMMA 12.

$$
\Phi(\tau) \equiv G_{5}(\tau)+11\left\{G_{4}(\tau)+2 G_{3}(\tau)+G_{2}(\tau)-1+2 g_{2}(\tau)+3 g_{3}(\tau)+g_{4}(\tau)+5 g_{5}(\tau)\right\} \quad\left(\bmod 11^{2}\right)
$$

This is proved in Appendix $\mathrm{C}$.

We use the symbol $(l, m)$ to denote an expression of the form

$$
11 \sum_{i=l}^{m-1} \lambda_{i} G_{i}(\tau)+\sum_{i=m}^{N} G_{i}(\tau),
$$


where $l<m \leqq N$ and the $\lambda_{i}$ are integral constants. Then in terms also of the notation of $\S 3.2$, we have (using Tables 5 and 6 )

$$
\Phi(\tau)(k, l, m) \equiv\left(l_{1}, m_{1}\right) \quad\left(\bmod 11^{2}\right)(m \geqq 7),
$$

where $m_{1}=m+5, l_{1}=\min (l+5, m-5)$.

Thus

$$
\begin{aligned}
& \Phi(\tau) G_{2}(11 \tau) \equiv(14,24) \quad\left(\bmod 11^{2}\right), \\
& \Phi(\tau) G_{n}(11 \tau) \equiv(9 n-4,9 n+6) \quad\left(\bmod 11^{2}\right)(n \geqq 3),
\end{aligned}
$$

by (12) and (14). Hence

$$
\pi\left(d_{n r}\right) \geqq 2 \text { if } r \leqq 9 n-5 .
$$

We can now prove the result complementary to Lemma 7. We have

LeMma 13. If $F(\tau) \in Y$, then $11^{-1} U\{\phi(\tau) F(\tau)\} \in X$.

Proof. We have, by (34),

$$
11^{-1} U \sum \mu_{n} 11^{\eta(n)} \phi(\tau) g_{n}(\tau)=\sum \sum \mu_{n} 11^{\eta(n)-3-\theta(n)+\theta(r)} d_{n r} g_{r}(\tau) .
$$

Thus we have to show that, for all $n$ and $r$,

$$
\eta(n)-3-\theta(n)+\theta(r)+\pi\left(d_{n r}\right) \geqq \xi(r) .
$$

Since $\pi\left(d_{n r}\right) \geqq 0$, (39) holds if $\theta(r)-\xi(r) \geqq \theta(n)-\eta(n)+3$. This is satisfied in the cases

$$
n \geqq 4, r \geqq n+10 ; n=2 \text { or } 3, r \geqq 12 .
$$

Also since $\pi\left(d_{n r}\right) \geqq \theta(n)-\theta(r)+2$, (39) holds if $\eta(n) \geqq \xi(r)+1$. This is satisfied in the cases

$$
n \geqq 4, r \leqq n ; \quad n=3, r=2 .
$$

Next, for $n \geqq 3$ and $n+1 \leqq r \leqq n+9$, we have $\pi\left(d_{n r}\right) \geqq 2$ and $\theta(r)-\xi(r) \geqq \theta(n)-\eta(n)+1$, which implies (39) for

$$
n \geqq 3, \quad n+1 \leqq r \leqq n+9 .
$$

Similarly we obtain

$$
n=2, \quad 4 \leqq r \leqq 11 .
$$

Finally we have by direct calculation $\pi\left(d_{33}\right)=3, \pi\left(d_{22}\right)=3, \pi\left(d_{23}\right)=4$, which give (39) for

$$
n=2, r=2 \text { and } 3 ; n=3, r=3 \text {. }
$$

Since (40) to (44) cover all integral $n, r$ with $n \geqq 2, r \geqq 2$, Lemma 13 is proved. We have also

LEMMA 14. If $F(\tau) \in Y^{0}$, then $11^{-1} U\{\phi(\tau) F(\tau)\} \in X^{0}$.

We have

$$
\lambda_{2}=\sum \mu_{n} 11^{\eta(n)-3-\theta(n)+2} d_{n 2}=\sum \sigma_{n}, \quad \text { say. }
$$

Now $\pi\left(d_{n 2}\right) \geqq \theta(n)-\theta(2)+2$, so that for $n \geqq 4, \eta(n) \geqq 2$, and so $\pi\left(\sigma_{n}\right) \geqq 1$. For $n=3$, 
$\pi\left(d_{32}\right)=4$ by direct calculation, and so $\pi\left(\sigma_{3}\right)=1$. Hence

$$
\lambda_{2} \equiv \mu_{2} 11^{-3} d_{22}(\bmod 11) \text {. }
$$

But $\pi\left(d_{22}\right)=3$, and hence if $\pi\left(\mu_{2}\right)=0$, then $\pi\left(\lambda_{2}\right)=0$. This proves the lemma.

\section{Proof of Theorem 1.}

Using the remark at the end of Lemma 11 we find

$$
L_{1}(\tau)=U \phi(\tau)=11 g_{2}(\tau)+2.11^{2} g_{3}(\tau)+11^{3} g_{4}(\tau)+11^{4} g_{5}(\tau)
$$

Hence $11^{-1} L_{1}(T) \in X^{0}$. It is now easily seen, by using the definition of $L_{n}(\tau)$ in (33) and Lemmas 8 and 14 , that

$$
11^{1-2 n} L_{2 n-1}(\tau) \in X^{0}, \quad 11^{-2 n} L_{2 n}(\tau) \in Y^{0} .
$$

This proves Lemma 10 and so Theorem 1 . In addition we see, as in $\S 3.5$, that Theorem 1 is best possible in the sense that

$$
p\left(l_{n}\right) \neq 0\left(\bmod 11^{n+1}\right)
$$

It is clear that the inductions used to prove Theorem 2 are dominated by the values of $\pi\left(c_{22}\right)$ and $\pi\left(d_{22}\right)$, in the sense that were either of these greater we could with greater effort establish a congruence modulo $11^{[3 n / 2]}$ or thereabouts. The actual computed values of $\pi\left(c_{n r}\right)$ and $\pi\left(d_{n r}\right)$ are much larger than those given by our inequalities as is shown by Tables 7 and 8 ; the difficult part of the induction, apart from " accidental " low values of $n$ and $r$, is when $r$ is close to $n$, and in fact it seems certain that $\pi\left(c_{n r}\right)$ and $\pi\left(d_{n r}\right)$ are about equal to $n$ in this case, not merely 3 or 4 as we prove. The introduction of a basis $g_{n}(\tau)$ with different orders of zeros at $\tau=i \infty$ is needed to cope with the case when $r<n$; the numbers $\theta(n)=6 n / 5$ which this involves are an inevitable and not wholly desirable complication. For $r \gg n$ Lehner's basis is equally satisfactory. Finally, the actual classes of functions $X, Y$ suffice for the induction, and are not best possible. We could use $\pi\left(d_{n r}\right) \geqq 1$ only, and a more elaborate form of Lemma 6 , plus a good deal of actual computation for low values of $n$ and $r$. This would avoid the appeal to Fine's equation, but the present method is shorter.

We may observe finally that, in comparison with $q=5$ and $q=7$, this proof is indeed "langweilig", as Watson suggested. In those cases, we can in effect deal directly with $U g^{n}(\tau)$ at $\tau=i \infty$, using the modular equation. In fact, his actual induction can be reduced $\dagger$ to about 2 pages each for $q=5$ and $q=7$, if it is expressed in terms of $\pi\left(c_{n r}\right)$ rather than fully written out formulae, by using explicit inequalities of the type $\pi\left(c_{n r}\right) \geqq[(5 n-r+1) / 2]$, for $q=5$. I think it likely that in the present case $q=11$ there exists an inequality

$$
\pi\left(c_{n r}\right) \geqq[(11 n-r+\delta) / 10],
$$

where $\delta=\delta(n, r)$ is small and of irregular behaviour, but I can at present see no technique for establishing this.

† See A. O. L. Atkin, Ramanujan congruences for $p_{k}(n)$; to appear in Canadian J. Math. 


\section{APPENDIX A}

Proof of Lemma 3. Following Newman [10], we define

$$
\sum_{n=0}^{\infty} p_{r}(n) x^{n}=f^{r}(x),
$$

where

$$
f(x)=\prod_{m=1}^{\infty}\left(1-x^{m}\right) .
$$

We shall in this appendix, where no confusion can arise, write $F(x)$ for the Fourier series of $F(\tau)$, with $x=e^{2 \pi i \tau}$. If now functions $g_{2}(x), g_{3}(x), G_{2}(x), G_{3}(x)$ are defined by

$$
\begin{aligned}
10 g_{2}(x) f^{5}(x) & =-\sum_{n=0}^{\infty}\left\{1+\left(\frac{n-3}{11}\right)\right\} p_{5}(n) x^{n}+11^{2} x^{25} f^{5}\left(x^{121}\right), \\
14\left\{g_{3}(x)+g_{2}(x)\right\} f^{7}(x) & =-\sum_{n=0}^{\infty}\left\{1+\left(\frac{2-n}{11}\right)\right\} p_{7}(n) x^{n}+11^{3} x^{35} f^{7}\left(x^{121}\right), \\
\left\{11^{2}+10 G_{2}(x)\right\} f^{5}\left(x^{11}\right) & =\sum_{n=-2}^{\infty} p_{5}(11 n+25) x^{n}, \\
\left\{11^{3}+14 G_{3}(x)+154 G_{2}(x)\right\} f^{7}\left(x^{11}\right) & =\sum_{n=-3}^{\infty} p_{7}(11 n+35) x^{n},
\end{aligned}
$$

it follows from (2.5.2), (2.7), and (2.8) of [10] that $G_{2}(\tau), G_{3}(\tau), g_{2}(\tau), g_{3}(\tau)$ belong to $S$, and that

$$
G_{2}(-1 / 11 \tau)=11^{2} g_{2}(\tau), \quad G_{3}(-1 / 11 \tau)=11^{3} g_{3}(\tau) .
$$

By examination of the actual expansions in Table 1 we see that in fact $G_{2}(\tau), G_{3}(\tau) \in S^{0}$ and $g_{2}(\tau), g_{3}(\tau) \in S^{\infty}$. We define next

$$
B(\tau)=G_{2}(\tau) g_{2}(\tau)-12
$$

$B(\tau)$ belongs to $S$, has a simple pole residue 1 at $\tau=0$ and $\tau=i \infty$, and satisfies

$$
B(\tau)=B(-1 / 11 \tau)
$$

Since $G_{3}(\tau) g_{3}(\tau)$ has the same properties, it follows that

$$
G_{3}(\tau) g_{3}(\tau)=B(\tau)+\text { constant }=B(\tau)+11 .
$$

We now define

$$
\left.\begin{array}{l}
G_{4}(\tau)=G_{2}^{2}(\tau)-11 G_{3}(\tau), \quad g_{4}(\tau)=g_{2}^{2}(\tau)-g_{3}(\tau), \\
G_{6}(\tau)=G_{2}(\tau) G_{4}(\tau), \quad g_{6}(\tau)=g_{2}(\tau) g_{4}(\tau), \\
G_{5}(\tau)=\eta^{12}(\tau) / \eta^{12}(11 \tau), \quad g_{5}(\tau)=\eta^{12}(11 \tau) / \eta^{12}(\tau) .
\end{array}\right\}
$$


That $G_{5}(\tau) \in S^{0}, g_{5}(\tau) \in S^{\infty}$ follows from Newman $[10,(2,3,3)]$. We have

$$
\left.\begin{array}{l}
G_{4}(-1 / 11 \tau)=11^{4} g_{4}(\tau), \\
G_{6}(-1 / 11 \tau)=11^{6} g_{6}(\tau), \\
G_{5}(-1 / 11 \tau)=11^{6} g_{5}(\tau) .
\end{array}\right\}
$$

Since $G_{5}(\tau)-G_{2}(\tau) G_{3}(\tau)+11 G_{4}(\tau)$ has a pole of order $m \leqq 1$ at $\tau=i \infty$, and is zero at $\tau=0$, it must be zero, since $\Gamma_{0}(11)$ has genus 1 . Hence

$$
G_{5}(\tau)=G_{2}(\tau) G_{3}(\tau)-11 G_{4}(\tau)
$$

We use this technique to derive the multiplication tables in Appendix B.

Next, we define inductively for $n \geqq 7$,

$$
G_{n}(\tau)=G_{n-5}(\tau) G_{5}(\tau), \quad g_{n}(\tau)=g_{n-5}(\tau) g_{5}(\tau)
$$

These results, together with the initial expansions in Table 1, establish the whole of Lemma 3 except for the assertions that the FS of $G_{n}(\tau), g_{n}(\tau)$ have integral coefficients (they clearly have rational coefficients from (46)). These can be proved in various ways, of which we choose the following. The functions $\alpha(\tau), \beta(\tau)$ of Fine [11, (3.20)], clearly have integral coefficients and belong to $S^{0}$. We thus can conclude that

$$
G_{2}(\tau)=\alpha(\tau), \quad G_{3}(\tau)=\beta(\tau)-3 \alpha(\tau),
$$

so that $G_{2}(\tau), G_{3}(\tau)$, and hence $G_{4}(\tau), G_{6}(\tau)$, have integral FS. It is also clear that $G_{5}(\tau)$ and $g_{5}(\tau)$ have integral FS. Now

$$
g_{2}(\tau)=g_{5}(\tau) G_{4}(\tau), \quad g_{3}(\tau)=g_{5}(\tau) G_{3}(\tau),
$$

so that $g_{2}(\tau), g_{3}(\tau)$, and hence $g_{4}(\tau), g_{6}(\tau)$ have integral FS. The result for all $n$ now follows from the definition (54).

\section{APPENDIX B \\ Fourier Series Expansions}

\section{Table 1}

With

$$
x=e^{2 \pi i \tau} \text { and } F(\tau)=\sum_{r=N}^{\infty} \alpha_{r} x^{r}
$$

we write

$$
F(\tau)=x^{N}\left(\alpha_{N}, \alpha_{N+1}, \alpha_{N+2}, \ldots\right)
$$


Then

$$
\begin{aligned}
& B(\tau)=x^{-1}(1,-5,17,46,116,252,533,1034,1961, \ldots), \\
& g_{2}(\tau)=x(1,5,19,63,185,502,1270,3046,6968,15335, \ldots) \\
& g_{3}(\tau)=x^{2}(1,9,49,214,800,2685,8274,23829,64843, \ldots) \\
& g_{4}(\tau)=x^{3}(1,14,102,561,2563,10285,37349,125290, \ldots) \\
& g_{5}(\tau)=x^{5}(1,12,90,520,2535,10908,42614,153960, \ldots) \\
& g_{6}(\tau)=x^{4}(1,19,191,1400,8373,43277,199982,844734, \ldots) \\
& G_{2}(\tau)=x^{-2}(1,2,-12,5,8,1,7,-11,10,-12, \ldots), \\
& G_{3}(\tau)=x^{-3}(1,-3,-5,24,-13,-22,13,-5,51, \ldots), \\
& G_{4}(\tau)=x^{-4}(1,-7,13,17,-84,57,93,-81,-63, \ldots) \\
& G_{5}(\tau)=x^{-5}(1,-12,54,-88,-99,540,-418,-648,594, \ldots), \\
& G_{6}(\tau)=x^{-6}(1,-5,-13,132,-233,-305,1404,-910,-1533, \ldots)
\end{aligned}
$$

Table 2

$$
\begin{array}{rlrl}
G_{4} & =G_{2}^{2}-11 G_{3}, & g_{4} & =g_{2}^{2}-g_{3}, \\
G_{5} & =G_{3} G_{2}-11 G_{4}, & 11 g_{5} & =g_{2} g_{3}-g_{4}, \\
G_{6} & =G_{2} G_{4}, & g_{6} & =g_{2} g_{4}, \\
G_{n+5} & =G_{n} G_{5}(n \geqq 2), \quad g_{n+5} & =g_{n} g_{5}(n \geqq 2) .
\end{array}
$$

Table 3

$$
\begin{array}{lll}
G_{2}(-1 / 11 \tau)=11^{2} g_{2}(\tau), & G_{2}(\tau)=x^{-2}+\ldots, & g_{2}(\tau)=x+\ldots, \\
G_{3}(-1 / 11 \tau)=11^{3} g_{3}(\tau), & G_{3}(\tau)=x^{-3}+\ldots, & g_{3}(\tau)=x^{2}+\ldots, \\
G_{4}(-1 / 11 \tau)=11^{4} g_{4}(\tau), & G_{4}(\tau)=x^{-4}+\ldots, & g_{4}(\tau)=x^{3}+\ldots, \\
G_{5}(-1 / 11 \tau)=11^{6} g_{5}(\tau), & G_{5}(\tau)=x^{-5}+\ldots, & g_{5}(\tau)=x^{5}+\ldots, \\
G_{6}(-1 / 11 \tau)=11^{6} g_{6}(\tau), & G_{6}(\tau)=x^{-6}+\ldots, & g_{6}(\tau)=x^{4}+\ldots
\end{array}
$$

Table 4

$$
\begin{array}{ll}
B G_{2}=11^{2}+G_{3}, & B g_{2}=1+11 g_{3}, \\
B G_{3}=11 G_{2}-G_{3}+G_{4}, & B g_{3}=g_{2}-g_{3}+11 g_{4}, \\
B G_{4}=11 G_{3}+G_{5}, & B g_{4}=g_{3}+11^{2} g_{5}, \\
B G_{5}=-12 G_{5}+G_{6}, & B g_{5}=-12 g_{5}+g_{6}, \\
B G_{6}=11^{2} G_{4}+11 G_{5}+G_{7} & B g_{6}=g_{4}+11 g_{5}+11^{2} g_{7} .
\end{array}
$$


A. O. L. ATKIN

Multiplication Table 5

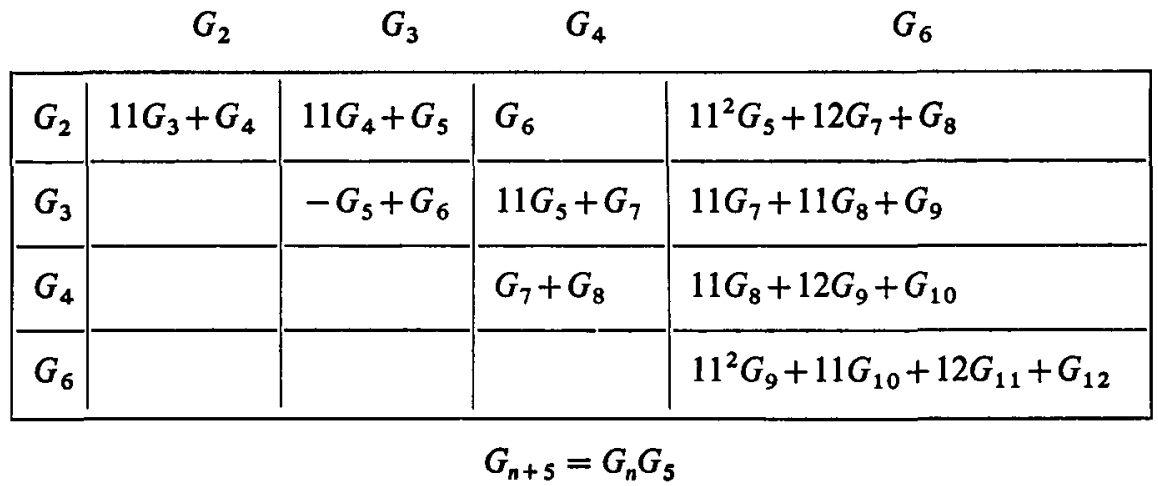

Multiplication Table 6

\begin{tabular}{|l|l|l|l|l|l|}
\multicolumn{2}{c}{$g_{2}$} & \multicolumn{2}{c}{$g_{3}$} & $g_{5}$ & \multicolumn{1}{c}{$g_{6}$} \\
\hline$G_{2}$ & $B+12$ & $1+11 g_{2}$ & $g_{2}+11 g_{3}$ & $g_{4}$ & $g_{3}+12 g_{4}+11^{2} g_{5}$ \\
$G_{3}$ & $G_{2}+11$ & $B+11$ & $1+11 g_{2}$ & $g_{3}$ & $g_{2}+11 g_{3}+11 g_{4}$ \\
$G_{4}$ & $G_{3}+G_{2}$ & $G_{2}+11$ & $B+12$ & $g_{2}$ & $1+12 g_{2}+11 g_{3}$ \\
$G_{5}$ & $G_{4}$ & $G_{3}$ & $G_{2}$ & 1 & $B+12$ \\
$G_{6}$ & $G_{5}+12 G_{4}+11 G_{3}$ & $G_{4}+11 G_{3}+11 G_{2}$ & $G_{3}+12 G_{2}+11^{2}$ & $B+12$ & $(B+12)^{2}$ \\
\hline
\end{tabular}

Tables 7 and 8 give the actual computed values of $\pi\left(\gamma_{n r}\right)$ and $\pi\left(\delta_{n r}\right)$ in

$$
U g_{n}(\tau)=\sum \gamma_{n r} g_{r}(\tau), \quad U\left\{\phi(\tau) g_{n}(\tau)\right\}=\sum \delta_{n r} g_{r}(\tau) .
$$

The calculations were performed modulo $11^{10}$, and $T$ stands for " $\geqq 10$ ".

Table 7. $\pi\left(\gamma_{n r}\right)$

\begin{tabular}{|c|c|c|c|c|c|c|c|c|c|}
\hline \multicolumn{2}{|c|}{$r=2$} & 3 & 4 & 5 & 6 & 7 & 8 & & 10 \\
\hline$n=2$ & 1 & 2 & 3 & 5 & 5 & 7 & 9 & 8 & $T$ \\
\hline 3 & 1 & 3 & 3 & 5 & 5 & 7 & 7 & 8 & $T$ \\
\hline 4 & 1 & 2 & 3 & 5 & 6 & 6 & 7 & 8 & $T$ \\
\hline 5 & 1 & 2 & 3 & 4 & 5 & 6 & 7 & 8 & $T$ \\
\hline 6 & 1 & 2 & 4 & 5 & 4 & 6 & 7 & 8 & $T$ \\
\hline 7 & 1 & 2 & 2 & 4 & 4 & 6 & 8 & 8 & $T$ \\
\hline 8 & 1 & 1 & 2 & 4 & 4 & 6 & 7 & 8 & $T$ \\
\hline 9 & 0 & 1 & 2 & 4 & 4 & 6 & 7 & 8 & $T$ \\
\hline 10 & 0 & 1 & 2 & 4 & 6 & 6 & 7 & 8 & $T$ \\
\hline
\end{tabular}


Table 8. $\pi\left(\delta_{n r}\right)$

\begin{tabular}{|c|c|c|c|c|c|c|c|c|c|}
\hline \multicolumn{2}{|c|}{$r=2$} & 3 & 4 & & 6 & 7 & 8 & & 10 \\
\hline$n=2$ & 1 & 2 & 2 & 4 & 4 & 7 & 7 & 9 & $T$ \\
\hline 3 & 1 & 1 & 2 & 5 & 4 & 6 & 7 & 8 & $T$ \\
\hline 4 & 0 & 1 & 2 & 4 & 4 & 6 & 7 & 8 & $T$ \\
\hline 5 & 0 & 1 & 2 & 4 & 4 & 6 & 7 & 8 & $T$ \\
\hline 6 & 0 & 1 & 3 & 4 & 5 & 6 & 7 & 8 & $T$ \\
\hline 7 & 0 & 1 & 2 & 4 & 4 & 6 & 8 & 8 & $T$ \\
\hline 8 & - & 1 & 2 & 4 & 4 & 6 & 7 & 9 & $T$ \\
\hline 9 & - & 1 & 2 & 4 & 4 & 6 & 7 & 8 & $T$ \\
\hline 10 & - & 1 & 2 & 3 & 4 & 6 & 7 & 9 & $T$ \\
\hline
\end{tabular}

Table 9

This table shows the relation of the notations of Lehner [6, 7], Fine [11], and Atkin and Hussain [12] to that of this paper.

\begin{tabular}{|l|l|l|l|}
\hline & \multicolumn{1}{|c|}{ Lehner } & Fine & Atkin and Hussian \\
\hline$B(\tau)$ & $A(\tau)-11$ & & \\
$g_{2}(\tau)$ & $C(\tau)$ & & \\
$g_{3}(\tau)$ & $D(\tau)-C(\tau)$ & & \\
$\phi(\tau)$ & $\Phi(\tau)$ & $u^{-1}(11 \tau)$ & \\
$G_{2}(\tau)$ & & $\alpha(\tau)$ & $-\lambda-13$ \\
$G_{3}(\tau)$ & & $\beta(\tau)-3 \alpha(\tau)$ & $-\mu+6 \lambda+16$ \\
$G_{5}(\tau)$ & & $v(\tau)$ & \\
$L_{n}(\tau)$ & $L\left(\tau ; 11^{\prime \prime}\right)$ & & \\
\hline
\end{tabular}

\section{APPENDIX C}

Proof of Lemma 12. The modular equation of degree 11 in $\Phi(\tau / 11)$ with coefficients in $S$ is given by Fine [11, (3.21)]. If we subject this to the transformation $\tau \rightarrow-1 / 11 \tau$, and observe that $\Phi(-1 / 121 \tau)=11 \phi(\tau)$, we obtain in our notation (the argument $\tau$ being omitted for brevity) 


$$
\begin{aligned}
g_{5}= & \phi\left(1+11 g_{2}+22 g_{3}+11 g_{4}\right)-\phi^{2}\left(11+99 g_{2}+88 g_{3}-11 g_{4}\right)+\phi^{3}\left(55+4.11^{2} g_{2}+2.11^{2} g_{3}\right) \\
& -\phi^{4}\left(11^{2}+12.11^{2} g_{2}+2.11^{2} g_{3}\right)-\phi^{5}\left(11^{2}-2.11^{3} g_{2}\right)+\phi^{6}\left(11^{3}-2.11^{3} g_{2}\right) \\
& -11^{4} \phi^{7}-11^{4} \phi^{8}+5.11^{4} \phi^{9}-11^{5} \phi^{10}+11^{5} \phi^{11} .
\end{aligned}
$$

Thus considering FS $\left(\bmod 11^{2}\right)$ we have

$$
g_{5} \equiv \phi\left(1+11 g_{2}+22 g_{3}+11 g_{4}\right)-\phi^{2}\left(11+99 g_{2}+88 g_{3}-11 g_{4}\right)+55 \phi^{3} \quad\left(\bmod 11^{2}\right)
$$

Now $\phi \equiv g_{5} \quad(\bmod 11)$ and hence

$$
\begin{aligned}
\phi & \equiv g_{5}-11\left(g_{7}+2 g_{8}+g_{9}-g_{10}+2 g_{12}+3 g_{13}+g_{14}+5 g_{15}\right) \quad\left(\bmod 11^{2}\right) \\
& =g_{5}-11 E, \quad \text { say. }
\end{aligned}
$$

Hence

$$
\Phi=\phi^{-1} \equiv G_{5}\left(1-11 G_{5} E\right)^{-1} \equiv G_{5}\left(1+11 G_{5} E\right)\left(\bmod 11^{2}\right),
$$

so that, by Table 6 ,

$$
\Phi \equiv G_{5}+11\left(G_{4}+2 G_{3}+G_{2}-1+2 g_{2}+3 g_{3}+g_{4}+5 g_{5}\right)\left(\bmod 11^{2}\right),
$$

which is Lemma 12.

\section{REFERENCES}

1. S. Ramanujan, Some properties of $p(n)$, the number of partitions of $n$, Proc. Cambridge Phil. Soc. 19 (1919), 207-210.

2. S. Ramanujan, Congruence properties of partitions, Math. Z. 9 (1921), 147-153.

3. J. M. Rushforth, Congruence properties of the partition function and associated functions, Proc. Cambridge Phil. Soc. 48 (1952), 402-413.

4. G. N. Watson, Ramanujans Vermutung über Zerfällungsanzahlen, J. Reine Angew. Math. 179 (1938), 97-128.

5. S. Chowla, Congruence properties of partitions, J. London Math. Soc. 9 (1934), 247.

6. J. Lehner, Ramanujan identities involving the partition function for the moduli $11^{\alpha}$, Amer.J. Math. 65 (1943), 492-520.

7. J. Lehner, Proof of Ramanujan's partition congruence for the modulus $11^{\mathrm{s}}$, Proc. Amer. Math. Soc. 1 (1950), 172-181.

8. J. Lehner, Divisibility properties of the Fourier coefficients of the modular invariant $j(\tau)$, Amer. J. Math. 71 (1949), 136-148.

9. M. Newman, Further identities and congruences for the coefficients of modular forms, Canadian J. Math. 10 (1958), 577-586. 313-320.

10. M. Newman, Remarks on some modular identities, Trans. Amer. Math. Soc. 73 (1952),

11. N. J. Fine, On a system of modular functions connected with the Ramanujan identities, Tohoku Math. J. 8 (1956), 149-164.

12. A. O. L. Atkin and S. M. Hussain, Some properties of partitions (2), Trans. Amer. Math. Soc. 89 (1958), 184-200.

The Atlas COMPUTER Laboratory Chilton, DidCOT 